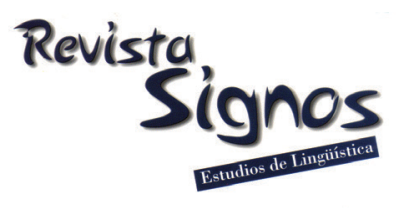

\title{
Definir y defender la identidad en un debate político: El caso de los blogueros emigrantes rusos
}

On defining and defending identity in political debates: A case-study of Russian immigrants bloggers

Nina Kresova

UniversidAd DE TARTU

EsTONIA

nkresova@ugr.es

\author{
Anna Ivanova \\ Universidad Autónoma de Chile \\ CHILE \\ anna.ivanova@uautonoma.cl
}

Recibido: 30-V-2013 / Aceptado: 20-I-2014

\section{Resumen}

El artículo presenta los resultados del estudio lingüístico de los posteos y comentarios publicados en los blogs de los emigrantes hablantes nativos de ruso sobre las elecciones presidenciales rusas de marzo de 2012. Mediante el análisis de las referencias deícticas 'nosotros', 'usted/-es/vosotros', 'ellos' y sus derivados, el estudio pretende detectar, clasificar y analizar el conjunto de pertenencias grupales (Turner, Hogg, Oakes, Reicher \& Wetherell, 1987; Baumeister \& Leary, 1995). El carácter novedoso del estudio radica en que se presta atención especial a la relación de los emigrantes con su contexto de origen. Asimismo, conviene resaltar la particularidad del material analizado ya que además de haber sido producido sin intervención del investigador, es producido en un entorno no interactivo. Los resultados muestran que para los blogueros del estudio es importante formar parte del grupo mayoritario ruso. Al mismo tiempo, tanto sus posteos como sus contribuciones en los comentarios revelan una identidad 'insegura' (Tajfel, 1974) que contrasta con la postura 'segura' y a veces hostil de los rusos residentes en Rusia.

Palabras Clave: Deixis personal, construcción discursiva de identidades, pertenencia, migración, blogging. 


\begin{abstract}
The present article deals with the corpus analysis of posts and comments published online by Russian emigrants about to presidential elections in 2012. By studying this corpus the paper intends to define, classify and analyze the notion of group belonging (Turner, Hogg, Oakes, Reicher \& Wetherell, 1987; Baumeister \& Leary, 1995). For the attainment of this goal, the paper departs from the analysis of deicitc personal references 'we', 'you' and 'they' and their derived forms. It is claimed that this approach draws attention to the way immigrants are related to their context of origin. The obtained results evidence the importance for immigrants to belong to a large group of Russians. At the same time, immigrants reveal a rather 'insecure' (Tajfel, 1974) position in their posts and especially comments, in comparison with 'secure' and sometimes hostile position of Russian residents authors.
\end{abstract}

Key Words: Person deixis, discursive construction of identity, belonging, migration, blogging.

\title{
INTRODUCCIÓN
}

El bienestar psíquico y físico del migrante de primera generación depende en gran medida de su capacidad para definir y asegurar su política identitaria en las nuevas circunstancias. Requiere encontrar su lugar en el complejo entramado de la sociedad de destino, pero también precisa renegociar su posición en la sociedad de origen. Las dos tareas son igualmente importantes y repercuten en el proceso de adaptación cultural y social del migrante así como en la convivencia en la sociedad de destino (Lewin, 1997; Phinney, Horenczyk, Liebkind \& Vedder, 2001).

El presente estudio se fundamenta en el análisis de los blogs de los emigrantes rusohablantes. Su objetivo general consiste en determinar los endo- y exogrupos aludidos en los discursos de los emigrantes sobre las pasadas elecciones presidenciales en Rusia, a través del análisis del uso de los pronombres 'nosotros', 'usted/-es/ vosotros', 'ellos' y sus derivados. Se tiene como objetivos específicos evaluar los grados de adhesión/enfrentamiento estableciendo la jerarquía de los grupos identificados (1), observar si se producen cambios en dicha jerarquía entre los posts y los comentarios de los emigrantes (2) y entre estos últimos y los comentarios de los rusos residentes en Rusia (3).

Los objetivos se han fijado teniendo en cuenta las siguientes premisas, que, a su vez, serán revisadas a lo largo de la investigación: a) los discursos de los emigrantes muestran identidades sociales 'inseguras' (Tajfel, 1974; de Fina, 2003; Yamaguchi, 2005; Krzyzanowsky \& Wodak, 2007; Clary-Lemon, 2010); b) los comentarios de un blog conforman un espacio en el que se negocian las identidades (Hevern, 2004); c) los blogueros emigrantes desean reafirmar su pertenencia a la cultura y la sociedad de origen, algo que les ayuda a conseguir su ciberdiario (Gorny, 2004). 
Para llevar a cabo esta investigación se adopta un enfoque propio de la lingüística de corpus para, en particular, localizar y examinar con detalle las referencias deícticas arriba indicadas. Desde una perspectiva similar, Wodak, Cillia, Reisigl y Liebhart (2009) y Fairclough (1989, 2000), entre otros, abordan el estudio de la identidad en el discurso político. En una línea muy similar, de Fina (2003), Yamaguchi (2005), Krzyzanowsky y Wodak (2007) y Clary-Lemon (2010) analizan las determinaciones sociales de los inmigrantes a través del uso de las referencias pronominales en sus entrevistas.

La novedad del presente estudio consiste en dirigir la atención a la relación del migrante con su entorno de origen. Por norma general, la investigación de las migraciones se desarrolla en los países receptores y su objeto suele ser la vida de los nuevos ciudadanos en el destino. No obstante, muchos psicólogos reivindican la importancia de la relación que mantiene el migrante con la cultura y la sociedad maternas para su integración en la sociedad receptora (Phinney et al., 2001; Padilla \& Pérez, 2003). Desde la antropología también se defiende la necesidad de llevar a cabo una etnografía multisituada para entender el fenómeno migratorio de manera holística (Marcus, 1995; Dietz \& Pérez, 2003; García, Álvarez \& Rubio, 2011).

Es también importante advertir el carácter novedoso que imprime a la investigación desarrollada el hecho de que se fundamente en el análisis de los discursos producidos en la Web 2.0. La red virtual colaborativa brinda a los científicos la posibilidad de estudiar al individuo y la sociedad sin necesidad de intervenir en el proceso de comunicación. Como es sabido, en los métodos etnográficos la presencia del investigador compromete el material obtenido y el alcance del posible sesgo resulta muy difícil de determinar (Hammersley \& Atkinson, 2007). Por otro lado, los datos producidos a partir de la Web 2.0 permiten acceder a una muestra más variada en términos geográficos, generacionales o de género. Si bien la investigación sobre la integración de la diáspora rusa suele centrarse en uno o dos países (Phinney et al., 2001; Remennick, 2002; Laitin, 2004; Morgunova, 2009), el material del presente estudio, en cambio, procede de los blogs de los emigrantes rusos residentes en once países, de distintas edades, profesiones y experiencia migratoria.

\section{La identidad social y las pertenencias grupales de los migrantes}

Tajfel define la identidad social como:

"that part of an individual's self-concept which derives from his knowledge of his membership of a social group (or groups) together with the emotional significance attached to that membership" (Tajfel, 1974: 69).

En el proceso de identificación se establecen distinciones endo- y exogrupales expresadas en el sentimiento de pertenencia (Tajfel, 1974; Tajfel \& Turner, 1979; 
Turner et al., 1987). El hecho de ser aceptado o, al contrario, rechazado, marginado por un colectivo empoderado repercute en la autoestima del individuo y sus relaciones con los demás (Baumeister \& Leary, 1995; Ellemers, Spears \& Doosje, 2002). Dentro de la misma lógica, un enemigo común aúna el grupo y lo hace sentirse más fuerte (Erikson, 1968; Turner et al., 1987).

La identidad social de un migrante sufre importantes transformaciones en el nuevo entorno (Padilla \& Pérez, 2003). Por regla general, el conjunto de pertenencias que tenía el individuo antes de su traslado a otro país no representa valor alguno para la sociedad de destino; sobre todo, si se trata de una migración hacia un lugar con mayor ranking político y económico. Titulaciones, experiencia laboral, contactos personales y profesionales ya no cuentan con el respaldo de los correspondientes grupos sociales, por lo que el recién llegado se enfrenta a la necesidad de definir y defender un nuevo conjunto de identificaciones sociales prácticamente desde cero.

Algunos estudios muestran que cuanto más inciertos sean los sentimientos de pertenencia de un individuo, mayor es su tendencia hacia el aislamiento y la marginación (Baumeister \& Leary, 1995; Lewin, 1997; Phinney et al., 2001). Es evidente que para que eso no ocurra, el migrante necesita fortalecer su posición dentro de la sociedad de destino. Pero tampoco se debe subestimar la importancia que tiene para él el respaldo de los endogrupos de la sociedad de origen (Lewin, 1997; Phinney et al., 2001; Schaafsma, 2011). Y es que estas pertenencias se transforman y se debilitan con la marcha del migrante. Si la pertenencia a un endogrupo, basado en el contacto presencial, todavía se sostiene gracias a diferentes vínculos personales (amistades, lazos familiares, relaciones laborales, vecindad, etc.), la relación con los endogrupos extensos -a nivel de etnia, nación, estado- se vuelve más frágil e inestable. Se trata de lo que Anderson (2006) llamó 'comunidades imaginadas'. La mayoría de sus miembros ni siquiera ha tenido contacto visual con el resto de integrantes, puede que tampoco hayan sabido nunca los unos de los otros, pero comparten una imagen mental de su comunión (Anderson, 2006). Los migrantes suelen encontrarse en la periferia de la 'comunidad imaginada' de su país de origen y, por tanto, son susceptibles de abandonar o ser excluidos del endogrupo.

\section{Construcción discursiva de la identidad}

Este trabajo asume que la identidad tiene carácter 'polifónico' (Barrett, 1999) y 'fluido' (Foucault, 1970; Butler, 1993), que es abierta, dinámica, contextualmente dependiente y que supone una construcción social en la que intervienen individuos, grupos e instituciones (de Fina, 2003; Bamberg, de Fina \& Schiffrin, 2011). Las prácticas discursivas son el escenario por excelencia para el ensayo, afirmación, negociación y modificación de identidades (Fairclough, 1989; Hall, 2000). En palabras de Hall (2000: 9), "identities are thus points of temporary attachment to the subject positions which discursive practices construct for us". 
Las identidades siempre son orientadas hacia una audiencia y pueden ser modificadas en función de su respuesta. Las narrativas monológicas, no interrumpidas por interlocutores, suelen presentar identidades mejor definidas y estables. La comunicación directa supone más negociación, más dinamismo en la identificación. Ambos modelos comunicativos se suceden en las redes sociales, lo cual las hace especialmente interesantes para el estudio de la construcción discursiva de las identidades.

\section{Migrantes y la web 2.0}

La importancia de la comunicación mediada por ordenador para la población especialmente vulnerable -como adolescentes, enfermos de cáncer, personas mayores o inmigradas- ha sido puesta de manifiesto en numerosos estudios (Helsper, 2008; Elias \& Lemish, 2009; Khvorostianov, Elias \& Nimrod, 2011; Mesch, 2012; Page, 2012).

En las últimas décadas el descenso de los precios del transporte aéreo, el desarrollo de la telefonía móvil y digital y, sobre todo, la expansión de Internet han hecho mucho más fluidas las relaciones de los que viven separados por la distancia. La web $2.0 \mathrm{y}$, en concreto, las redes sociales pasaron a desempeñar un rol especial en la vida de los migrantes. Las utilizan para difundir y encontrar información que los ayuda a establecerse en la nueva sociedad, para compartir sus vivencias, conocer gente del nuevo entorno y mantenerse en contacto con gente de su lugar de origen.

Las comunicaciones que mantienen los migrantes con los distintos grupos sociales de los países de procedencia y de destino juegan un papel muy importante en el desarrollo de sus procesos de identificación. Sus nuevas o renovadas identidades se construyen "in keeping with the diversity and dynamics of the ongoing adjustment to a new society and maintenance of their original cultural identity" (Elias \& Lemish, 2009: 535). A diferencia de las redes como Facebook o Twitter, donde los usuarios multilingües suelen publicar entradas en diferentes idiomas, los blogs generalmente tienen una lengua que predomina sobre las demás. Su elección, en el caso de los diarios de los migrantes, a menudo deja entrever las prioridades del autor: estrechar lazos con los representantes del país de destino o mantener y reforzar vínculos con la gente que comparte su cultura de origen. Esta segunda opción es la escogida por los autores de las ciberbitácoras exploradas en esta investigación.

\section{Material de estudio}

La muestra analizada procede de la base de datos de blogs de los emigrantes rusohablantes ${ }^{1}$. La búsqueda inicial se llevó a cabo usando una serie de palabras clave en varias plataformas (Blogspot, Blogger, Wordpress y LiveJournal) y a través del buscador Google de $b \operatorname{logs}{ }^{2}$. Posteriormente la lista se fue engrosando siguiendo el método 'bola de nieve' (Baltar \& Brunet, 2012). 
Los resultados de las búsquedas iniciales mostraron la casi absoluta preferencia de los blogueros rusohablantes por la comunidad social Livejournal (LJ). Dicha red fue la primera en ofrecer este tipo de servicio a los usuarios de Internet y a pesar de haber sido creada fuera de las fronteras rusas, rápidamente fue 'colonizada' por los internautas rusos (Gorny, 2004; Alexanyan \& Koltsova, 2009; Morgunova, 2012).

Los blogs o weblogs son "frequently modified web pages in which dated entries are listed in reverse chronological sequence" (Herring, Scheidt, Bonus \& Wright, 2005: 142). En el estudio se analizan los Personal Journals o diarios personales, uno de los cuatro géneros que Herring et al. (2005) distinguen en los blogs. Su contenido comprende narrativas sobre la vida del bloguero así como sus pensamientos, que pueden ser comentadas por otros usuarios. Lo que diferencia los blogs de las redes sociales como Facebook es su mayor jerarquización de los contenidos. En un diario personal el autor tiene potestad para proponer y desarrollar temas, gestionar el acceso a sus publicaciones y administrar aportaciones de otras personas.

Los tres corpus analizados se compusieron a partir de una selección temática y cronológica. Se revisaron los 72 blogs de la mencionada base de datos entre el 1 y el 10 de marzo de 2012 en busca de entradas referentes a las elecciones presidenciales rusas del 4 de marzo de 2012. Se registraron 43 posts de 17 autores que conformaron el primer corpus del presente estudio (6931 palabras). Los comentarios de estas entradas fueron separados entre los realizados por emigrantes (2do corpus) y los residentes en países rusohablantes ( $3^{\text {er }}$ corpus). De dicha distinción resultaron dos corpus de 18143 y 31616 palabras respectivamente. Los comentarios proceden de 170 usuarios emigrantes y 420 usuarios no emigrantes.

Como se observa en la Tabla 1, los 17 blogueros del estudio provienen de 11 países. La franja de edad se sitúa entre los 30 y 60 años. 5 autores llevan entre 1 y 10 años en el extranjero, 7 de ellos emigraron hace más de 10 años y 5 hace más de 20 años. Son 4 mujeres y 13 hombres los sujetos que componen la muestra, en la que los hombres publicaron un mayor número de entradas y recibieron más comentarios. El más popular fue un bloguero residente en Reino Unido, periodista de profesión, que en 7 posts obtuvo 651 comentarios. 
Tabla 1. Datos sobre los autores y los posts seleccionados para el análisis.

\begin{tabular}{|l|l|l|l|l|l|}
\hline A & B & C & D & E & F \\
\hline M & 31 & Gran Bretaña & 8 & 7 & 651 \\
\hline M & 43 & Bélgica & 22 & 8 & 165 \\
\hline M & 51 & Estados Unidos & 16 & 1 & 154 \\
\hline M & 35 & Australia & 7 & 3 & 138 \\
\hline M & 41 & Canadá & 10 & 6 & 89 \\
\hline M & 37 & Israel & 22 & 2 & 80 \\
\hline M & 31 & Nueva Zelanda & 8 & 2 & 67 \\
\hline M & 41 & Canadá & 11 & 2 & 60 \\
\hline M & 43 & Estados Unidos & 19 & 1 & 57 \\
\hline F & 37 & Estados Unidos & 16 & 1 & 43 \\
\hline M & 64 & Estados Unidos & 24 & 1 & 37 \\
\hline M & 30 & España & 4 & 2 & 30 \\
\hline F & 42 & Italia & 20 & 1 & 12 \\
\hline F & $55-60$ & Finlandia & $0-5$ & 3 & 8 \\
\hline M & 36 & Alemania & 11 & 1 & 5 \\
\hline M & Sin datos & Estados Unidos & 12 & 1 & 4 \\
\hline F & $55-60$ & Estados Unidos & 36 & 1 & 2 \\
\hline
\end{tabular}

Leyenda: A: sexo del bloguero (M-masculino, F-femenino); B: su edad; C: país de residencia; D: años de residencia en el extranjero; E: número de entradas publicadas sobre las elecciones; F: número total de comentarios obtenidos.

Entre los que comentan las entradas suele haber personas que conocen al autor en el mundo real, si bien la mayoría son seguidores. Los autores también comentan sus propios posts aunque en este estudio sus réplicas fueron tratadas indistintamente dentro del segundo corpus. Se observa que los blogs de los emigrantes atraen la atención de otros emigrantes residentes en el mismo país o zona geográfica, como Australia y Nueva Zelanda. El tema de las elecciones presidenciales suscitó un interés especial y algunos posts subieron al top-list diario de LJ de modo que llegaron a un público mayor del habitual. Es por eso que ciertas entradas del presente estudio llegaron a recibir hasta 350 comentarios procedentes de una gran variedad de lectores. La notable mayor cantidad de comentaristas residentes en los países rusohablantes se debe en este caso al tema elegido, pues en otros estudios se había registrado mucha mayor interacción entre los blogueros emigrantes y baja participación de los no emigrantes (Kresova, 2013a; Kresova, 2013b).

El tópico de las últimas elecciones presidenciales en Rusia fue escogido debido a que es un acontecimiento que atañe a todos los ciudadanos rusos y que, al someterse a debate, obliga a definir las posturas políticas y sociales de los interlocutores. Cabe resaltar que estos últimos comicios despertaron un interés inusual en la comunidad rusa y la participación entre los emigrantes fue susceptiblemente mayor que en las ediciones anteriores ${ }^{3}$. Debido a ello, el material escogido presenta un magnífico 
escenario para observar las adherencias endo- y exogrupales de los blogueros emigrantes.

\section{Deixis y su aplicación en los estudios de discurso}

El término deixis se refiere a "the way speakers orient themselves and their listeners in terms of person, time and space in relation to the immediate situation of speaking” (McCarthy \& Carter, 1994: 178). Es una herramienta lingüística de 'orientación' que permite ubicar al remitente en relación con el destinatario de su mensaje (Simpson, 1993). Palabras como 'aquí' y 'allí', 'ahora' y 'después', 'nuestro' y ‘suyo' cobran significado en cada situación particular, por lo tanto, no pueden ser comprendidas fuera del contexto de su emisión.

Las referencias deícticas se dividen en espaciales, temporales y personales (Fillmore, 1997). Estas últimas definen la identidad de los interlocutores en una situación comunicativa al establecer diferencias entre el hablante, el destinatario y los demás (Fillmore, 1997; Trask, 1999).

La exploración de las referencias deícticas en cuanto señas identitarias tiene una larga tradición en los estudios del discurso. Los pronombres personales según Levinson en particular cifran "the social identities of participants or the social relationships between them, or between one of them and persons and entities referred to" (de Fina, 2003: 52).

La deixis personal puede ser una poderosa herramienta en el discurso político, que sirve tanto para fines de persuasión como de manipulación (Zupnik, 1994; Wodak et al., 2009; Arroyo, 2000; Fairclough, 2000; Ivanova, 2012). Wodak et al. (2009: 45-47) elaboraron una detallada descripción de las potencialidades semánticas del pronombre 'nosotros', ampliando la tradicional distinción entre "addressee-inclusive and addresseeexclusive 'we', and between a speaker-inclusive and speaker-exclusive 'we"'. El examen del uso de este pronombre en los discursos de los políticos, medios de comunicación y en grupos de discusión les permitió detectar y describir una serie de estrategias para la construcción de la identidad nacional austriaca. Los autores recurrieron asimismo a la codificación del material analizado por grupos de referencia (Wodak et al., 2009), procedimiento que se ha utilizado también en este estudio.

El uso de los pronombres personales en las narrativas de los migrantes fue objeto de estudio en de Fina (2003), Yamaguchi (2005), Krzyzanowsky y Wodak (2007) o ClaryLemon (2010). De Fina ha examinado las entrevistas de los inmigrantes mexicanos en los Estados Unidos. En particular, presta su atención a cómo los narradores gestionan su rol individual en el relato de su trayectoria migratoria. El uso de los pronombres 'yo', 'tú/Usted' y 'nosotros' le ha permitido observar el nivel de agencia que se asignan los entrevistados. Con todo esto, de Fina (2003: 76) llega a la conclusión de que "these Mexican immigrants spontaneously orient towards collective subjectivity when asked about individual experiences". 
Yamaguchi (2005) analiza el uso de los pronombres 'yo', 'tú', 'nosotros' y 'ellos' en las entrevistas de dos japoneses. Su microanálisis de discursos situados pone de manifiesto que los entrevistados, que crecieron y se educaron en Estados Unidos, presentan una identidad nacional múltiple, ambivalente y contextualmente dependiente.

Krzyzanowsky y Wodak (2007) llegan a conclusiones similares al examinar las referencias deícticas personales en los discursos procedentes de 45 grupos de discusión organizados en ocho países europeos. El análisis de las representaciones colectivas e individuales de los migrantes de primera, segunda y tercera generación sugiere que:

"migrant belongings are always perceived as having 'too much... or too little' identification and never reach 'a proper fit' of origin and target communities” (Krzyzanowsky \& Wodak, 2007: 114).

Utilizando el marco teórico elaborado en Wodak et al. (2009), Clary-Lemon (2010) emplea el análisis del uso de los pronombres 'nosotros' y 'ellos' para observar las estrategias discursivas de asimilación/inclusión, singularización y disimilación/ exclusión en las entrevistas de los migrantes irlandeses en Canadá. En la tesis final de su trabajo se argumenta que:

"the contingent and multiple nature of immigrant identity as one that is hybrid, caught between 'we/us' and 'they/them', 'self' and 'other', 'home' and 'away'” (Clary-Lemon, 2010: 17).

El presente estudio también aborda el análisis de los pronombres personales para observar las pertenencias grupales expresadas en los discursos de los migrantes. A diferencia de las investigaciones reseñadas arriba, hace uso de una metodología cuantitativa que permite ver y comparar la importancia estadística de los grupos semánticos detectados para cada corpus.

Por otra parte, el presente estudio examina los discursos que se producen sin la intermediación de un entrevistador. Los autores del blog son siempre propulsores de la situación comunicativa y conservan cierta potestad sobre los demás participantes. Por lo tanto, hablan de lo que ellos mismos quieren hablar y con los que ellos desean hablar. En este sentido, las narrativas producidas en los blogs son más próximas a las naturalmente ocurridas que las obtenidas con ayuda del método etnográfico.

Por último, el material del estudio permite comparar el uso de los pronombres personales en un contexto monológico (posts) y en interacción directa (comentarios). Sin ser equiparables por completo, el post se acerca a las narrativas procedentes de las entrevistas tradicionales y el comentario tiene su símil en una réplica originada en los grupos de discusión. Tanto uno como otro material han sido objeto de análisis en los trabajos reseñados arriba, sin embargo, este estudio también explota la posibilidad de su comparación. 


\section{Metodología aplicada}

La investigación se llevó a cabo mediante: 1) búsqueda de posts relativos al tema elegido; 2) compilación de un corpus común; 3) detección de todas las formas de pronombres personales 'nosotros', 'Usted/-es/vosotros' y 'ellos'; 4) identificación de los patrones semánticos en el corpus común; 5 . análisis comparado de sus ocurrencias en los tres corpus por separado.

En la realización del presente trabajo se recurrió al software WordSmith@ Tools v. 6. Con su ayuda se obtuvo una lista de frecuencia de palabras en la que se detectaron todas las formas de uso de los pronombres 'nosotros', 'Usted/-es/vosotros' y 'ellos'. A continuación se determinaron las concordancias de cada término en el corpus común. Las líneas de concordancias incluyeron cinco palabras a la izquierda y a la derecha de cada término y se ordenaron teniendo en cuenta sus funciones sintácticas y posición en el corpus. Dicho método dio como resultado "a list of all the occurrences of a particular search term in a corpus, presented within the context that they occur in" (Baker, 2006: 71). El paso siguiente fue llevar a cabo una codificación y clasificación paralela de todas las ocurrencias de las referencias deícticas localizadas previamente. Durante el proceso de codificación se aplicó el análisis de colocaciones de todos los términos "which show the associations and connotations they have, and therefore the assumptions which they embody" (Stubbs, 1996 citado por Baker, 2006: 96). Después de una primera codificación, que arrojó un alto índice de variaciones, se realizó un segundo análisis cruzado del que resultaron ocho grupos semánticos:

1. Gente en general;

2. todos los rusos;

3. rusos que viven en Rusia;

4. emigrantes;

5. distintos grupos sociales (partidarios de una ideología política, seguidores de diferentes partidos y líderes, autoridades locales y nacionales, etc.);

6. nivel privado (familia, amigos, conocidos);

7. seres vivos no humanos;

8. seres inertes.

Tras determinar dichos grupos semánticos, se procedió a realizar un análisis cuantitativo, comparando los resultados obtenidos para cada conjunto de referencias deícticas (1. 'nosotros' / 2. 'Usted/-es/vosotros' / 3. 'ellos') en los tres corpus. A continuación se presentan los resultados de este análisis. 


\section{Resultados}

La lectura comparada de los datos obtenidos de los corpus de los emigrantes y los no emigrantes muestra que los últimos emplean el pronombre 'nosotros' y sus derivados con más frecuencia. En sus comentarios el uso de dichas referencias conforma el 0,67\% de todo el vocabulario ${ }^{5}$. En los posts de los emigrantes el mismo dato alcanza el $0,61 \%$, pero en los comentarios baja hasta $0,50 \%$.

Si el campo de referencia más aludido por los emigrantes, tanto en los posts como en los comentarios, es el entorno privado del hablante (familias, amigos, conocidos), los no emigrantes, en primer lugar, se sienten integrantes de la comunidad de rusos en general y, en segundo lugar, nombran el grupo de rusos que residen en Rusia. Después del ámbito privado, los posts de los emigrantes revelan su pertenencia a la comunidad de rusos en general. Sin embargo, en los comentarios este campo de referencia se reduce drásticamente y su lugar pasa a ocupar el grupo de los expatriados. Tanto los emigrantes como los no emigrantes emplean el 'nosotros' al hablar de distintos grupos sociales, sobre todo al definir sus afiliaciones políticas, si bien es cierto que se detecta un ligero descenso de este tipo de identificaciones en los comentarios de los emigrantes respecto a sus posts. El Gráfico 1 ilustra lo dicho hasta ahora.

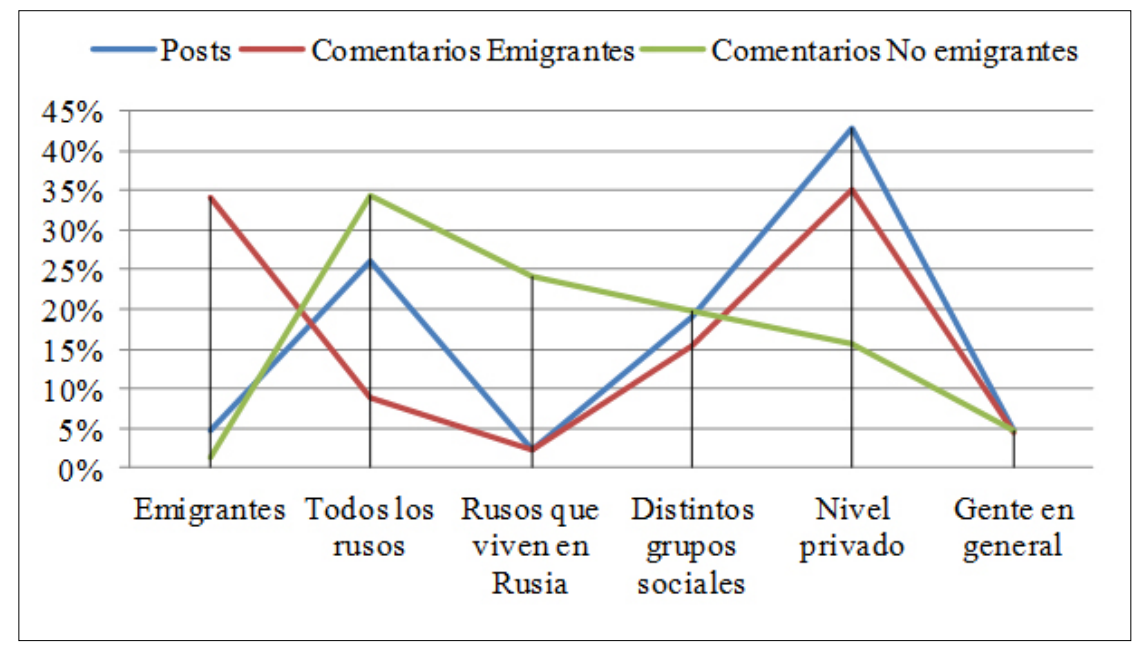

Fuente: Elaboración propia

Gráfico 1. Distribución por grupos semánticos. 'Nosotros' y sus derivados. ${ }^{6}$

Al observar las distintas formas casuales del pronombre 'nosotros', destaca la acumulación del lexema 'nas' que coincide en genitivo, acusativo y preposicional en el campo referencial 'emigrantes'. Mayoritariamente se emplea para situar la acción explicando algo que ha sucedido en el lugar donde vive el emigrante ('u nas'). Así, en este grupo se encontraron 7 unidades en nominativo y las 21 formas casuales antes indicadas. Asimismo, cabe advertir que en comparación con los posts, en los comentarios se amplía el uso de formas posesivas en ámbitos distintos del privado. 
Si se analizan las concordancias del pronombre 'nosotros' en nominativo, se detecta que en los comentarios de los no emigrantes existe una tendencia hacia un mayor uso de verbos transitivos: $49 \%$ frente al $36 \%$ de los comentarios de los emigrantes. De por sí, los verbos transitivos poseen mayor capacidad resolutiva, definiendo acciones sobre objetos/sujetos concretos. Pero además, entre los usados por los no emigrantes predominan verbos de procesos materiales mientras que los emigrantes aluden más a procesos relacionales y mentales (Halliday, 1994). Así las cosas, si los primeros 'eligen', 'aprenden', 'respetan', 'cambian', 'aplastan', 'pierden', 'ganan', 'compran' y 'venden', los segundos 'se enteran de', 'ven', 'disputan', y 'escuchan'.

Los emigrantes usan todas las formas de 'vy' (Usted/vosotros/Ustedes) con menos frecuencia que los no emigrantes (Gráfico 2). Además, no son habituales en los posts $(0,38 \%)$, que al poseer un carácter más monológico dejan poco espacio para las apelaciones directas. Su número sube en los comentarios, donde los emigrantes los usan casi con la mitad de frecuencia que los no emigrantes $(0,51 \%$ frente a $0,93 \%)$.

En los tres corpus, el primer campo de referencia ha sido el de nivel privado seguido de distintos grupos sociales. Se trata de apelaciones a otro u otros participantes del diálogo a título personal o en calidad de representantes de un grupo de afiliaciones ideológicas. Tras los dos campos de referencia citados, los no emigrantes a menudo emplean 'vy' para referirse a todos los expatriados mientras que los emigrantes apenas lo hacen para dirigirse al grupo de 'los rusos que residen en Rusia'.

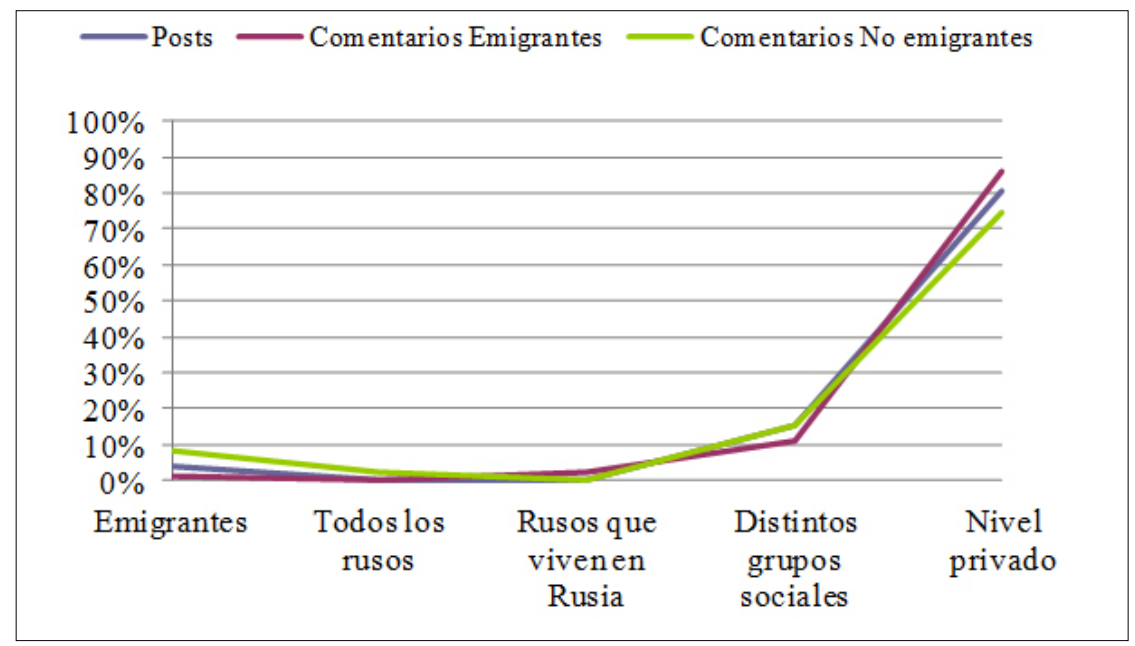

Fuente: Elaboración propia

Gráfico 2. Distribución por grupos semánticos. 'Vy’ y sus derivados.

Como era de esperar, en los discursos sobre el tema electoral el campo de referencia más usado para todas las formas del pronombre 'ellos' ha sido el de distintos grupos sociales (Gráfico 3). Son 'ellos' los partidarios de un candidato, de un partido político, gente de un determinado tipo de vida, representantes del poder gubernamental, etc. 
Los emigrantes en sus comentarios apelan con más claridad a un 'Otro' con perfil sociopolítico. En sus posts también es notable la presencia de los grupos de 'rusos que viven en Rusia' y 'rusos en general' aunados bajo el pronombre 'ellos'. No obstante, dicha distinción cae en desuso en sus comentarios.

Por el contrario, cerca del $20 \%$ de todas las formas del pronombre 'ellos' empleadas por los rusos residentes en Rusia se refiere a los emigrantes. Por último, huelga advertir que, como en el caso del 'nosotros', los no emigrantes atribuyen a 'ellos' un mayor número de acciones expresadas con verbos transitivos de procesos materiales.

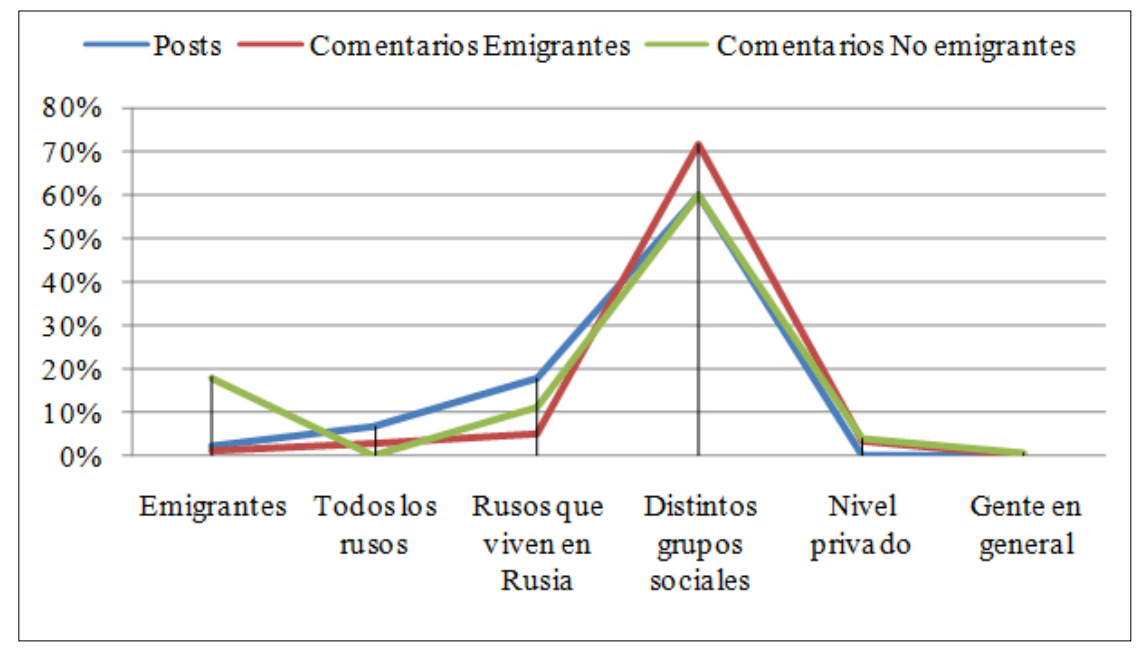

Fuente: Elaboración propia

Gráfico 3. Distribución por grupos semánticos. 'Ellos’ y sus derivados.

\section{Discusión}

Los resultados muestran que los blogueros emigrantes de este estudio proceden con mucha cautela a la hora de utilizar 'nosotros', inclinándose por pertenencias fundamentadas en vínculos personales. Consideraciones similares se encuentran en el trabajo de de Fina (2003), aunque a diferencia de los mexicanos recién llegados a Estados Unidos los emigrantes rusos proceden con muchas reservas en su relación con otros rusos residentes en el extranjero.

Entre estos últimos hay 'ellos' que los blogueros ven como un exogrupo. Son los que votaron a un determinado candidato o los que, como constata el siguiente fragmento, se olvidaron de sus raíces:

$\mathrm{CE}^{7}$ : El hombre ha de vivir ahí donde quiere vivir. Pero olvidarse de sus raíces y llamar a Rusia "rashka": eso me parece rastrero. Y hay muchos emigrantes que después de abandonar el país enseguida dejan de identificarse con él y tratan de olvidarse de su ciudadanía como si fuera algo vergonzoso. 
En cambio, los no emigrantes no tienen reparos para decir 'nosotros' refiriéndose al grupo étnico, pueblo o estado rusos. Ellos emplean expresiones como 'nuestro país' (4 veces), 'nuestro pueblo' (2) y 'nuestra sociedad' (2), que no aparecen en los posts y los comentarios de los emigrantes.

CNE: Claro, es normal que una persona se preocupe por las demás. Pero Usted no dirá que "nos han vuelto a disparar" refiriéndose, por ejemplo, a lo ocurrido esta mañana y mediodía en Asdod [...] Sin embargo, el autor [del post] escribe "estamos jugando con un fullero", ¿comprende?, "NOSOTROS". ¿Qué "nosotros" si son "ellos"?, por mucho que eso les hiera.

En este ejemplo el comentarista manifiesta su convicción de que los emigrantes están excluidos del grupo nacional de los rusos. Otra mujer no emigrante sale a defender a los expatriados y su polémica se desarrolla a lo largo de otros 19 comentarios. Se les suma un bloguero más, también residente en Rusia, pero los propios emigrantes no interceden en la discusión.

Es importante subrayar que la definición de 'nosotros' y de 'ellos' cambia de los posts a los comentarios de los emigrantes. Analizando los blogs se debe recordar que a la hora de componer el post, su autor tiene muy presente la imagen de sus lectores potenciales. Se dirige a ellos figurando las respuestas posibles, si bien sabe que al escribir en un diario público (abierto a todos los usuarios registrados e incluso a los anónimos ${ }^{8}$ ), su texto puede producir efectos inesperados. Con todo, el autor se siente más seguro escribiendo el post que comentándolo. Por ello, los blogueros emigrantes definen allí sus pertenencias con más seguridad. En el espacio dialógico de los comentarios, se vuelven aún más prudentes y evitan confrontaciones abiertas.

Un bloguero emigrante hace en su post el llamamiento a la unión y la tolerancia de los conciudadanos.

PE: Gente, ¿os habéis vuelto locos? Bueno, Putin ha ganado, ¿y qué? [...] Pero ¿por qué os peleáis como si fuerais perros rabiosos? Somos ciudadanos del mismo país.

Esta entrada tiene 117 palabras entre las que hay tres referencias al 'nosotros' con el significado 'todos los rusos'. En los comentarios escritos por el autor del post, que comprenden 1371 palabras, hay solo cuatro formas del mismo pronombre. Tres de ellas pertenecen al grupo semántico de 'todos los rusos' y uno corresponde al de 'emigrantes'.

En los comentarios los no emigrantes le dicen varias veces al autor que 'es fácil dar esos consejos desde el país de los duendes'. Al principio el bloguero responde con bromas ('vente y también estarás bien'), pero poco a poco se ve en la necesidad de defender su posición: 
CPE: Vete a Japón, vive y trabaja allí durante 6 años, luego búscate un trabajo en Australia, arregla toda la documentación necesaria, trasládate. Entonces veremos lo tolerante y sereno que vas a estar...

Al final resulta que el autor del post invierte más tiempo en defender su derecho a 'dar consejos' y hablar de parte de todos los rusos que en desarrollar el argumento de su post.

El hecho de evitar confrontaciones con los no emigrantes debe de ser la razón por la que los emigrantes usan mucho menos las apelaciones directas con 'vosotros/ ustedes/Usted'. En los comentarios también hubo rusos residentes en Rusia que arremetieron duramente contra los emigrantes. He aquí uno de los fragmentos más representativos:

CNE1 -los mierda-emigrantes en contra de Putin. Cojonudo. Y los ciudadanos rusos, por Putin.

CNE2 -estupideces... Yo vivo en Rusia y ninguno de mis amigos o conocidos votó a Putin... qué más da si el hombre emigró o no... siento vergüenza por los ciudadanos rusos tan estrechos de mente como Usted.

CNE1 -sí... pero siente vergüenza por nuestros emigrantes que vierten inmundicias sobre nuestra patria. La mayoría absoluta votó a Putin. Respete la elección de la mayoría.

CPE -vamos a aclararlo una vez más. Putin no es igual a la Patria. Y aquí nadie vierte inmundicias sobre la Patria, créame.

El mismo guión se repite en todos los casos de confrontación entre los no emigrantes y los emigrantes que se han detectado en los tres corpus. Los emigrantes no levantan el guante: generalmente responden con silencio o tratan de apaciguar los ánimos. Los que salen en su defensa son otros no emigrantes, que a veces se ven envueltos en largos debates sobre el asunto.

Entre 'ellos' generalmente están los 'Otros', los que son distintos de nosotros, con los que nos comparamos, a los que admiramos y respetamos, reprobamos y tememos. Son necesarios para fijar nuestra postura, definir nuestras características para nosotros mismos y para los demás (Erikson, 1968; Turner et al., 1987).

Para algunos de los no emigrantes los que abandonaron la patria pasaron al bando opositor de los 'Otros'. Los emigrantes, sin embargo, no quieren levantar paredes entre ellos y los rusos que residen en Rusia. Y aquí hay que resaltar que en los comentarios los emigrantes se distancian aún menos de los demás rusos. Tanto los unos como los otros se alinean con algunos bloques políticos. Pero sólo a los emigrantes el hecho de formar parte de un grupo de partidarios políticos junto con los rusos no emigrantes les permite sentirse más in. Puede que por eso -y en consonancia con la tendencia arriba indicada de no distanciarse de los demás rusos- los emigrantes en 
sus comentarios hablen activamente de 'ellos': gobernadores, funcionarios, votantes de un candidato, etc.

En los comentarios de los emigrantes se observa un intenso proceso de negociación de identidades. Predomina el estilo de negociación colaborativa pero también se registra pérdida de agencia y estilo evasivo. El hecho de que los blogs estén escritos en ruso lleva a la conclusión de que sus autores buscan contacto con gente que tiene la misma lengua nativa y semejante bagaje sociocultural. Un bloguero emigrante entrevistado por Gorny (2004: 36-37) explicaba así los motivos que le indujeron a abrir un diario en LJ:

"For me, as for a Russian person living abroad, LJ is a possibility to maintain contact with my habitual linguistic and social environment. During the years that I lived abroad in the absence of the Internet and LJ in particular, I started having problems with the Russian language, as well as with my social and national identification. My circle of daily contacts was almost exclusively francophone, and I could not fully identify myself with this circle, which produced a feeling of social discomfort. With the advent of the home Internet and LJ I have found again my place in the circle of the Russian intellectual class with which I feel a deep affinity. I feel that these people need me, and our interaction serves as something important beyond us."

El presente estudio confirma la visión de este bloguero. Los emigrantes persisten en mantener una comunicación abierta con sus conciudadanos, lo cual quiere decir que es algo que necesitan, algo que les permite sentirse mejor. De no ser así, simplemente escribirían e-mails a sus contactos directos y no irían a votar, no debatirían asuntos políticos de Rusia, no firmarían peticiones, etc. Los emigrantes de esta investigación buscan ser parte de los grandes endogrupos de su patria, evitan irritar a los empoderados (discursivamente hablando) dentro de este grupo, ceden en las discusiones, moderan su lenguaje. Ellos quieren gustar a sus lectores y esperan sus comentarios. Pero se encuentran con que el grupo mayoritario -representado por los individuos que están seguros de su pertenencia, de ser miembros de pleno derechocuestiona su inclusión (Krzyzanowsky \& Wodak, 2007: 114).

\section{CONCLUSIÓN}

Los discursos de los blogueros emigrantes de este estudio reflejan una identidad social 'insegura'. Su sentido de pertenencia a los grupos mayoritarios de su país de origen es incierto y contextualmente dependiente. Lo dicho se observa claramente al contrastar el uso de los pronombres personales por parte de los emigrantes con los comentarios de los residentes en Rusia. El examen comparado de los posts y los comentarios de los emigrantes también revela una considerable pérdida de confianza a la hora de expresar afiliaciones o discrepancias en la conversación virtual. 
Los emigrantes rusos de la muestra presentan un comportamiento similar al de los japoneses y los irlandeses entrevistados por Yamaguchi (2005) y Clary-Lemon (2010). Junto con otras investigaciones (de Fina, 2003; Krzyzanowsky \& Wodak, 2007), los resultados del estudio sugieren que el carácter inseguro de la identidad social y del conjunto de pertenencias a colectivos mayoritarios es propio de los migrantes de primera generación procedentes de diferentes culturas, distintas edades y experiencia migratoria. Sin embargo, en contra de la propuesta de Yamaguchi (2005) y ClaryLemon (2010), que atribuyen esta particularidad a la naturaleza híbrida de la identidad de los migrantes (Bhabha, 2003), las conclusiones extraídas invitan a pensar en subjetividades nómadas (Braidotti, 1994), entre otras razones por no haber observado "the emergence of an 'interstitial' agency that refuses the binary representation of social antagonism" (Bhabha, 2003: 58), pero sí una fluctuación entre distintas identificaciones y "simultaneous occurrence of many of these at once" (Braidotti, 1994: 4).

Esta investigación incide en la relación del migrante con su país de origen y demuestra su importancia para los expatriados de la muestra. Krzyzanowsky y Wodak, 2007: 115) definen al migrante como aquel que "having left home but not having arrived (in a primarily abstract sense)". Los discursos analizados en esta investigación evidencian que los blogueros emigrantes rusohablantes tampoco terminan de abandonar su país de nacimiento y buscan afirmar sus pertenencias, empoderar su identidad social en las narrativas y los discursos de su ciberbitácora. 


\section{REFERENCIAS BIBLIOGRÁFICAS}

Alexanyan, K. \& Koltsova, O. (2009). Blogging in Russia is not Russian. En A. Russell \& N. Echchaibi (Eds.), Blogging. International blogging: Identity, politics, and networked publics (pp. 65-84). Bern: Peter Lang.

Anderson, B. (2006). Imagined communities: Reflections on the origin and spread of nationalism. Londres/Nueva York: Verso.

Arroyo, J. (2000). Mire usted Sr. González ... Personal deixis in Spanish politicalelectoral debate. Journal of Pragmatics, 32(1), 1-27.

Baker, P. (2006). Using corpora in Discourse Analysis. Londres/Nueva York: Continuum.

Baltar, F. \& Brunet, I. (2012). Social research 2.0: Virtual snowball sampling method using Facebook. Internet Research, 22(1), 57-74.

Bamberg, M., de Fina, A. \& Schiffrin, D. (2011). Discourse and identity construction. En S. J. Schwartz, K. Luyckx \& V. L. Vignoles (Eds.), Handbook of Identity Theory and Research (pp. 177-199). Nueva York: Springer.

Barrett, R. (1999). Indexing polyphonous identity in the speech of African American drag queens. En M. Bucholtz, A. Liang \& L. Sutton (Eds.), Reiventing identitities (pp. 313-332). Oxford: Oxford University Press.

Baumeister, R. F. \& Leary, M. R. (1995). The need to belong: Desire for interpersonal attachments as a fundamental human motivation. Psychological Bulletin, 117, 497-529.

Bhabha, H. K. (2003). Culture's in-between. En S. Hall \& P. du Gay (Eds.), Question of Cultural Identity (pp. 53-60). Londres: Sage.

Braidotti, R. (1994). Nomadic subjects. Embodiment and sexual difference in contemporary feminist theory. Nueva York: Columbia University Press.

Butler, J. (1993). Bodies that matter. Londres: Routledge.

Clary-Lemon, J. (2010). 'We're not ethnic, we're Irish!': Oral histories and the discursive construction of immigrant identity. Discourse \& Society, 21(1), 5-25.

De Fina, A. (2003). Identity in narrative: A study of immigrant discourse. Amsterdam: John Benjamins.

Dietz, G. \& Pérez Galán, B. (Eds.). (2003). Globalización, resistencia y negociación en América Latina. Madrid: Catarata.

Elias, N. \& Lemish, D. (2009). Spinning the web of identity: The roles of the internet in the lives of immigrant adolescents. New Media Society, 11, 533-551. 
Ellemers, N., Spears, R. \& Doosje, B. (2002). Self and social identity. Annual Review of Psychology, 53, 161-186.

Erikson, E. H. (1968). Identity, youth and crisis. Nueva York: W. W. Norton Company.

Fairclough, N. (1989). Language and Power. Londres: Longman.

Fairclough, N. (2000). New labour, new language? Londres: Routledge.

Fillmore, C. J. (1997). Lectures on deixis. Stanford: CSLI Publications.

Foucault, M. (1970). The order of things: An archeology of the human sciences. Londres: Tavistock Publications.

Foucault, M. (1984). The history of sexuality. Harmondsworth: Penguin.

García Castaño, F. J., Álvarez Veinguer, A. \& Rubio Gómez, M. (2011). Prismas trasescalares en el estudio de las migraciones. Revista de Antropología Social, 20, 203-228.

Gorny, E. (2004). Russian LiveJournal: National specifics in the development of a virtual community [en línea]. Disponible en: Russian-cyberspace.org http://www. ruhr-uni-bochum.de/russ-cyb/library/texts/en/gorny_rlj.pdf.

Gorny, E. (2006). A creative history of the Russian internet. Tesis de doctorado, Goldsmiths College, Universidad de Londres, Inglaterra.

Hall, S. (2000).Who needs identity? En P. Du Gay, J. Evans \& P. Redman (Eds.), Identity: A reader (pp. 15-30). Londres: Sage Publications and the Open University.

Halliday, M. A. K. (1994). An introduction to functional grammar. Londres: Edward Arnold.

Hammersley, M. \& Atkinson, P. (2007). Oral accounts and the role of interviewing. Ethnography: Principles in Practice (pp. 97-120). Londres: Routledge.

Helsper, E. (2008) Digital inclusion: An analysis of social disadvantage and the information society. Londres: Department for Communities and Local Government.

Herring, S. C., Scheidt, L. A., Bonus, S. \& Wright, E. (2005). Weblogs as a bridging genre. Information, Technology \& People, 18(2), 142-171.

Hevern, V. W. (2004) Threaded identity in cyberspace: Weblogs \& positioning in the dialogical self. Identity: An International Journal of Theory and Research, 4(4), 321335.

Ivanova, A. (2012) Barack Obama's Presidential Governing on the Internet: Web 2.0 and the Pervasiveness of Political Language. Tesis de doctorado, Universidad de Sevilla, España. 
Khvorostianov, N., Elias, N. \& Nimrod, G. (2011). Without it I am nothing: The internet in the lives of older immigrants. New Media Society, 14(4), 583-599.

Kresova, N. (2013a). Friends and enemies of Russian emigrants: discursive strategies to reinforce social identity. Ponencia presentada en el BAAL-CUP Seminar "Narrative inquiry in transnational migratory contexts: Epistemological and methodological issues", University of Southampton, Southampton, Reino Unido.

Kresova, N. (2013b). Mi vida aquí: Narrativas en los blogs de los emigrantes rusos. Ponencia presentada en la VII International Conference on Interdisciplinary Social Sciences, Charles University, Praga, República Checa.

Krzyzanowsky, M. \& Wodak, R. (2007). Multiple identities, migration, and belonging: Voices of migrants'. En C. Caldas-Couthard \& R. Iedema (Eds.), Identity Troubles (pp. 95-119). Londres: Palgrave Macmillan.

Laitin, D. (2004). The De-cosmopolitanization of the Russian Diaspora: A view from Brooklyn in the "Far Abroad". Diaspora, 13, 5-35.

Lewin, K. (1997). Psycho-sociological problems of a minority group. En K. Lewin (Ed.), Resolving social conflicts and field theory in social science (pp. 107-115). Washington, DC: American Psychological Association.

Marcus, G. (1995). Ethnography in/of the World System: The emergence of MultiSited Ethnography. Annual Review of Anthropology, 24, 95-117.

McCarthy, M. \& Carter, R. (1994). Language as discourse: Perspectives for language teaching. Londres/Nueva York: Longman.

Mesch, G. S. (2012). Minority status and the use of computer-mediated communication: A test of the social diversification hypothesis. Communication Research, 39, $317-$ 337.

Morgunova, O. (2009). Russkoe prisutstvie v Britanii. Moskva: Sovremennaia Ekonómika i Pravo.

Morgunova, O. (2012). National living on-line? Some aspects of the Russophone e-diaspora map [en línea]. Disponible en: http://www.e-diasporas.fr/working-papers/ Morgunova-Russophones-EN.pdf

Padilla, A. M. \& Pérez, W. (2003). Acculturation, social Identity, and social cognition: A new perspective. Hispanic Journal of Behavioral Sciences, 25, 35-55

Page, R. E. (2012). Narratives of illness and personal blogs. En R. Page (Ed.), Stories and Social Media: Identities and Interaction (pp. 49-65). Nueva York: Routledge. 
Phinney, J., Horenczyk, G., Liebkind, K. \& Vedder, P. (2001). Ethnic identity, immigration, and well-being: An interactional perspective. Journal of Social Issues, 3(57), 493-510.

Remennick, L. (2002). Transnational community in the making: Russian-Jewish immigrants of the 1990s in Israel. Journal of Ethnic and Migration Studies, 28(3), 515-530.

Schaafsma, J. (2011). Discrimination and subjective well-being: The moderating roles of identification with the heritage group and the host majority group. European Journal of Social Psychology, 41(6), 786-795.

Simpson, P. (1993). Language, ideology and point of view. Londres/ Nueva York: Routledge.

Tajfel, H. \& Turner, J. C. (1979). An integrative Theory of Intergroup Conflict. En W. G. Austin \& S. Worchel (Eds.), The Social Psychology of Intergroup Relations (pp. 33-47). Monterey, CA: Brooks-Cole.

Tajfel, H. (1974). Social identity and intergroup behaviour. Social Science Information, 13, 65-93.

Trask, R. L. (1999). Key concepts in language and linguistics. Londres: Routledge.

Turner, J. C., Hogg, M. A., Oakes, P. J., Reicher, S. D. \& Wetherell, M. S. (1987). Rediscovering the social group: A self-categorization theory. Oxford, UK: Basil Blackwell.

Wodak, R., de Cillia, R., Reisigl, M. \& Liebhart, K. (2009). The discursive construction of national identity. Edinburgh: Edinburgh University Press.

Yamaguchi, M. (2005). Discursive representation and enactment of national identities: The case of Generation 1.5 Japanese. Discourse \& Society, 16(2), 269-299.

Zupnik, Y.-J. (1994). A pragmatic analysis of the use of person deixis in political discourse. Journal of Pragmatics, 21, 339-383. 


\section{NOTAS}

1 http://www.vitartis.com. La base ha sido elaborada por Nina Kresova en el marco del proyecto "Storytelling on Web 2.0: The case of migrants' personal blogs" financiado con los fondos de los programas ERMOS 52 (Marie Curie COFUND) y ETF 8874 (Estonian Research Council).

2 http://www.google.es/blogsearch

3 Así, el consulado de Rusia en Bonn ha anunciado en su página web que el número de votantes en los comicios de 2012 fue seis veces mayor que en las elecciones anteriores. Consultar: http:// ruskonsulatbonn.de/ru/news/index.php/2012/03/05/o-vyborax-prezidenta-rossiiskoi-federacii4-marta-2012-goda.html (v. 20/11/2012). Los blogueros hicieron hincapié en las colas que se formaron el día de las elecciones en los puntos habilitados para la votación en el extranjero. En total, a las urnas acudió el $65 \%$ de los votantes censados.

4 El sistema de pronombres personales del ruso tiene 6 formas en singular (ia [yo], ty [tú], Vy [Usted], on [él], oná [ella] y onó [ello]) y 3 formas en plural [my [nosotros/as], vy [vosotros/as] y oní [ellos/ as]]. Presenta cambios de género, número así como la declinación. En este estudio también se han tenido en cuenta formas de pronombres posesivos. En total, se han detectado y analizado 35 formas pronominales diferentes.

5 El porcentaje que conforma el uso de todas las formas de 'nosotros' registradas en el corpus respecto al volumen total de todas las palabras contenidas en el mismo corpus. De esta forma, si en los comentarios de los no emigrantes hay 212 pronombres 'nosotros' y sus derivados, este número conforma el 0,67\% de las $31.616(100 \%)$ lexemas empleadas.

6 En este caso el porcentaje se calcula respecto al número total de los pronombres 'nosotros' y sus derivados registrados en el corpus correspondiente.

7 Las siglas utilizadas en los fragmentos citados corresponden a: P (post), C (comentario), E (emigrante), N (no emigrante). Así, para referirse al emigrante autor del post se usan las siglas CPE. Para distinguir a los comentaristas, a las siglas CE o CNE se añade un número que indique el orden de intervención.

8 Esta característica es regulada por el bloguero. Pero la mayoría de los blogs analizados están configurados para ser abiertos a cualquier internauta. 